have done as well with these two letters as with the others if they had differed from each other sufficiently to emphasize their difference rather than their similarity. In my own test types, the letter $\mathrm{C}$ is never mistaken for any other letter. Even so, Dr. Owen made no mistake with $\mathrm{C}$ at 8.50 metres, and only one mistake with $O$. They are clearly good letters. Moreover, they are letters with which children are most familiar. They cannot possibly be left out of any useful set of test types.

Drs. Hartridge and Owen have chosen what they call " letters of medium difficulty." The reason for the choice does not appear, but the more simple a test the greater its accuracy and value as an instrument of precision, and the more simple the letters the better our test types will be for the purpose intended.

As far as the shape of the letters is concerned, there seems to be little or nothing to choose between Snellen's $5 \times 5$ and Green's $4 \times 5$ on the score of visibility, with the possible exception of $\mathrm{O}$, which would be more easily read when round than when oval.

There are many other points of interest in the paper, but I have already trespassed sufficiently upon your valuable space.

SHEFFIELD. Yours truly,

Percival J. Hai.

\title{
GERMAN OPHTHALMOLOGICAL LITERATURE
}

To the Editor of The British Journal of Ophthalmology

SIR,-In view of the letter in your last issue from Julius Springer, the following facts may be of interest. Towards the end of last year, I had occasion to order from Berlin the numbers of the Jahresbericht which have been published since the war broke out, viz. : Vols. XLIV and XIVII. The price paid was as stated in the publisher's letter, 40s. for Vol. XLIV, 32s. for Vol. XLVII.

Last week I received by post a second copy of Vol. XLVII, with a bill enclosed for $£ 1$ 1s. 6 d.

Your annotation, Sir, seems to have had the effect of reducing the price of this work by a considerable amount. I can offer no suggestion as to why I have received a second copy which I did not ask for. It may be a subtle form of humour on the publisher's part; he may be under the impression that I wrote the annotation in question; allow me to state that such is not the case, and to inform him through the medium of your pages that he may have his spare copy by instructing his representative in this country to call at my address on any morning between the hours of 9.30 and 11.30 a.m.

46, Wimpole Street, W.1.

Yours faithfully,

R. R. JAMEs. 